\title{
Erratum: FoxO1 integrates direct and indirect effects of insulin on hepatic glucose production and glucose utilization
}

InSug O-Sullivan, Wenwei Zhang, David H. Wasserman, Chong Wee Liew, Jonathan Liu, Jihye Paik, Ronald A. DePinho, Donna Beer Stolz, C. Ronald Kahn, Michael W. Schwartz \& Terry G. Unterman

Nature Communications 6:7079 doi: 10.1038/ncomms8079 (2015); Published 12 May 2015; Updated 24 Jul 2015

This Article contains errors in Figs 1 and 6, the latter of which was introduced during the production process. In Fig. 1a, the western blot lanes should have been labelled ' -+-+-+-+ '. In Fig. 6, the lower of each pair of labels indicating hepatic glucose production or utilization should read 'hgu' or 'HGU'. The correct versions of both figures appear below. 
a

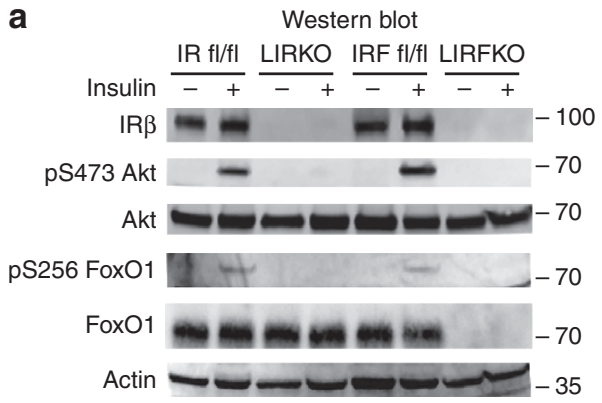

C

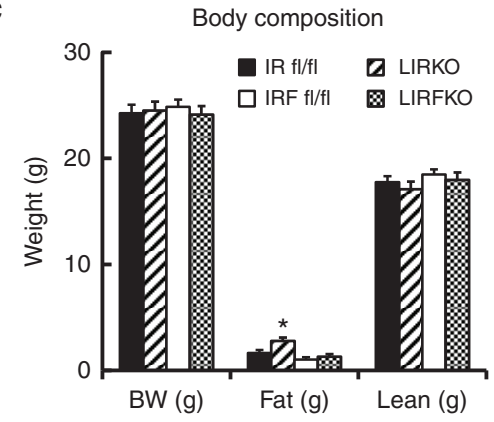

e

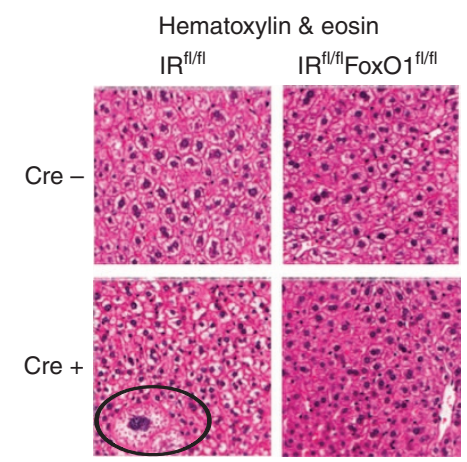

b

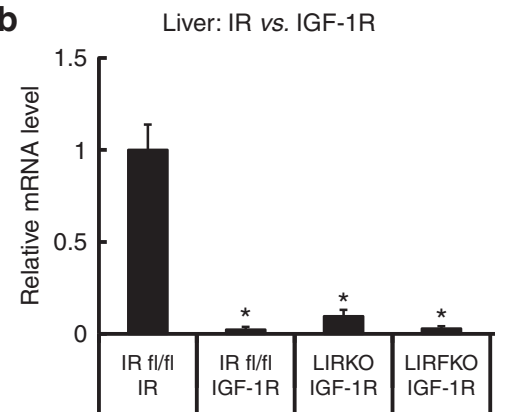

d

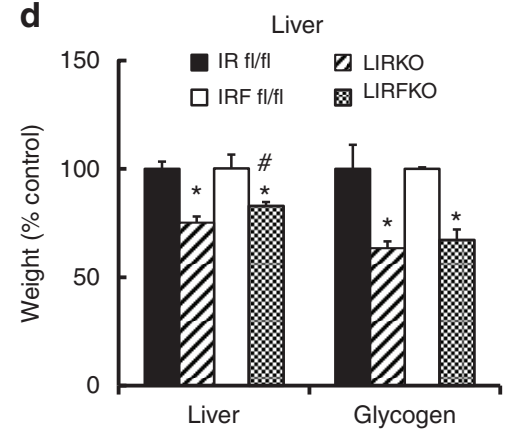

f
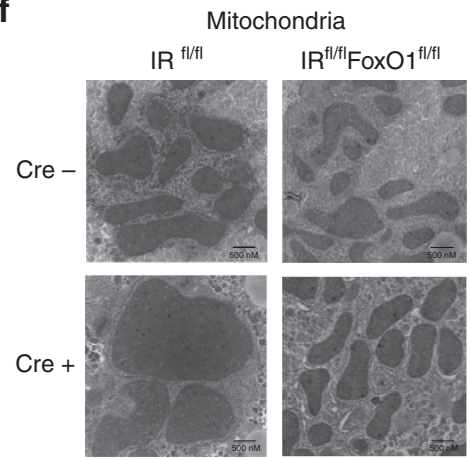

Figure 1 
a

Fasting

Insulin
$\vdots$
IR

b

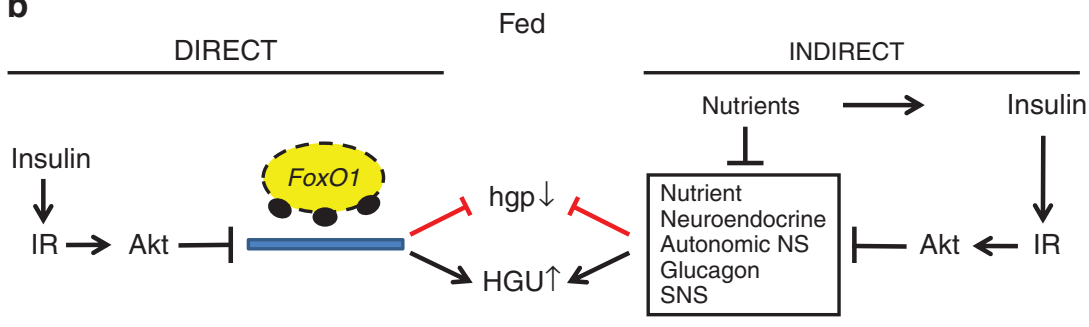

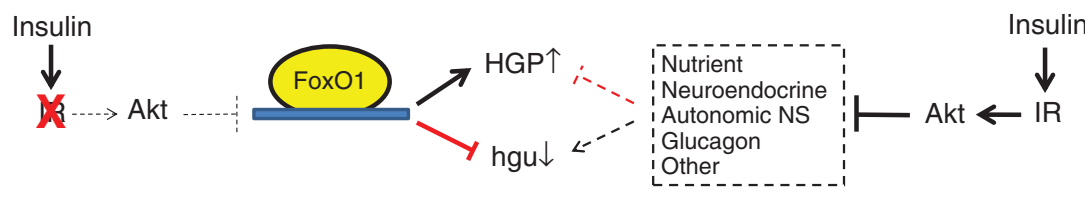

$\downarrow$

Figure 6 